\title{
Total non-alcoholic beverage consumption in 12-18 year olds from two urban Irish secondary schools
}

\author{
S. Millar, B. McNulty and A. McKevitt \\ School of Agriculture and Food Science, University College Dublin, Dublin, Republic of Ireland
}

Recent attention from health organisations has highlighted the consumption of sugar sweetened beverages among adolescents, and their impact on health ${ }^{(1,2)}$. Within Ireland, there is a lack of current beverage consumption data among this age group, the most recent data on this cohort being from the National Teens' Food Survey (NTFS) 2005-2006 ${ }^{(3)}$.

The objectives of the present pilot study were: 1) to assess current total non-alcoholic beverage consumption in a sample of $12-18$ year old adolescents from two mixed-sex, demographically distinct, urban Irish secondary schools; 2) to assess if beverage consumption differed between the two schools; and 3) to assess if patterns of consumption had changed over the last 10 years.

Ethical approval was received from the Human Research Ethics Committee at University College Dublin. An anonymised, selfadministered beverage consumption survey was administered to students from two secondary schools in the age range of $12-18$ years. One school was classified as disadvantaged through the Department of Education and Skills' DEIS scheme. The second school was a private fee-paying school. Significant differences were found in sex and age distribution between the two schools $(p<0 \cdot 001)$. Thus, linear regression, adjusting for age and sex, was used to detect between school differences in beverage consumption frequencies.

The total study population was 441 (DEIS $n=213$; Private $n=228$ ). Median age was 14 years and $49 \%$ of the study population were male. Self-reported median beverage intakes (g/day) of the 6 most highly consumed beverages are presented in Figure 1, differences in quantity of water, tea, fizzy drinks and diet fizzy drinks consumed became apparent when compared to the NTFS. Significant differences in beverage consumption between the two schools are presented in Figure 2, no differences between schools were noted for fruit juice or milk consumption.

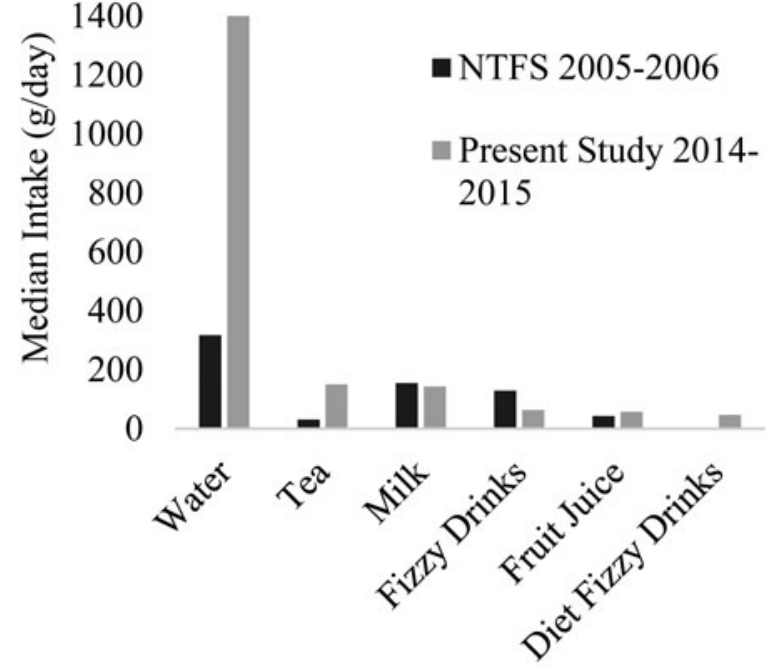

Fig. 1. Median beverage intakes from the present study (2014-2015) and the NTFS (2005-2006)

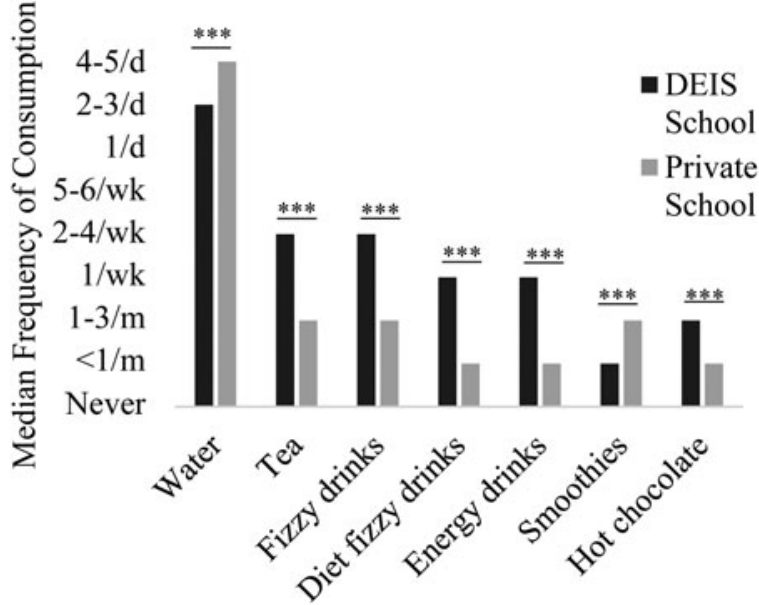

Fig. 2. Between school differences in beverage consumption. *** $\mathrm{p}<0.001$. Abbreviations: $d$, day; wk, week; $m$, month

Despite the use of self-reported data from adolescents and a non-validated survey, this cross-sectional study provides a snapshot of current beverage intakes among adolescents, which merits further investigation. Overall, these findings suggest a positive trend in beverage consumption among adolescents, but indicates a clear distinction in intake between a private and a DEIS school.

1. World Health Organisation (2015) Guideline: Sugars intake for adults and children.

2. Scientific Advisory Committee on Nutrition (2014) Draft Carbohydrate and Health Report.

3. Irish Universities Nutrition Alliance (2006) National Teens' Food Survey. 